MATHEMATICS OF COMPUTATION

Volume 75, Number 254, Pages 847-857

S 0025-5718(06)01871-0

Article electronically published on January 3, 2006

\title{
A NEW SUPERCONVERGENT COLLOCATION METHOD FOR EIGENVALUE PROBLEMS
}

\author{
REKHA P. KULKARNI
}

\begin{abstract}
Here we propose a new method based on projections for the approximate solution of eigenvalue problems. For an integral operator with a smooth kernel, using an interpolatory projection at Gauss points onto the space of (discontinuous) piecewise polynomials of degree $\leq r-1$, we show that the proposed method exhibits an error of the order of $4 r$ for eigenvalue approximation and of the order of $3 r$ for spectral subspace approximation. In the case of a simple eigenvalue, we show that by using an iteration technique, an eigenvector approximation of the order $4 r$ can be obtained. This improves upon the order $2 r$ for eigenvalue approximation in the collocation/iterated collocation method and the orders $r$ and $2 r$ for spectral subspace approximation in the collocation method and the iterated collocation method, respectively. We illustrate this improvement in the order of convergence by numerical examples.
\end{abstract}

\section{INTRODUCTION}

Consider the eigenvalue problem

$$
T \phi=\lambda \phi,
$$

where $T$ is a compact linear operator defined on a complex Banach space. A standard technique for solving (1.1) approximately is to replace $T$ by a finite rank operator. The approximate solution of (1.1) is then obtained by essentially solving a matrix eigenvalue problem. If $\pi_{n}$ is a sequence of finite rank projection operators converging to the identity operator pointwise, then in the classical Galerkin method $T$ is replaced by $T_{n}^{G}=\pi_{n} T \pi_{n}$, and in the iterated Galerkin method proposed by Sloan, $T$ is replaced by $T_{n}^{S}=T \pi_{n}$. When $\pi_{n}$ is an interpolatory projection, the choice of $T_{n}^{C}=\pi_{n} T \pi_{n}$ gives rise to a collocation method, whereas $T_{n}^{S}=T \pi_{n}$ is associated with the iterated collocation method. These methods are extensively studied in literature. (See, for example [1, [3, 4], [5, [6, [7, 10.)

We propose to approximate $T$ by the finite rank operator

$$
T_{n}^{M}=\pi_{n} T \pi_{n}+\pi_{n} T\left(I-\pi_{n}\right)+\left(I-\pi_{n}\right) T \pi_{n} .
$$

Then $\left\|T-T_{n}^{M}\right\|=\left\|\left(I-\pi_{n}\right) T\left(I-\pi_{n}\right)\right\| \rightarrow 0$ as $n \rightarrow \infty$.

In this paper an integral operator

$$
T u(s)=\int_{0}^{1} k(s, t) u(t) d t, \quad s \in[0,1]
$$

Received by the editor March 2, 2003 and, in revised form, October 28, 2004.

2000 Mathematics Subject Classification. Primary 47A10, 47A58, 47A75, 65J99, 65R20.

Key words and phrases. Eigenvalue, spectral subspace, integral equations, collocation, Gauss points.

(C)2006 American Mathematical Society Reverts to public domain 28 years from publication 
with a smooth kernel is considered. Note that $T: C[0,1] \rightarrow C[0,1]$ is compact.

Consider a partition of $[0,1]$ with $n$ subintervals and norm $h$. The collocation points are chosen to be the $n r$ Gauss points, obtained by shifting $r$ Gauss points in $[-1,1]$ to each of the subintervals of the partition. Hence the interpolation points do not include the end points of the subintervals, i.e., the partition points. As a consequence, the interpolating piecewise polynomial is discontinuous at the partition points. Let $X_{n}$ be the space of all (discontinuous) piecewise polynomials of degree $\leq r-1$ with respect to the above partition of $[0,1]$. For $f \in C[0,1]$, let $\pi_{n} f$ be the unique element of $X_{n}$ which interpolates $f$ at the $n r$ collocation points. By using a result of [2], $\pi_{n}$ can be extended to $L^{\infty}[0,1]$, and then $\pi_{n}: L^{\infty}[0,1] \rightarrow X_{n}$ is a projection.

Let $\lambda$ be a nonzero eigenvalue of $T$ with algebraic multiplicity $m$ and let $P$ be the associated spectral projection. Let $\hat{\lambda}_{n}^{M}$ and $\hat{\lambda}_{n}^{C}=\hat{\lambda}_{n}^{S}$ denote the arithmetic mean of the $m$ eigenvalues of $T_{n}^{M}$ and $T_{n}^{C}$ (or $T_{n}^{S}$ ), respectively, which approximate $\lambda$. Let $P_{n}^{M}, P_{n}^{C}$ and $P_{n}^{S}$ denote the spectral projections associated with the group of $m$ eigenvalues of $T_{n}^{M}, T_{n}^{C}$ and $T_{n}^{S}$, respectively. We prove that

$$
\left|\lambda-\hat{\lambda}_{n}^{M}\right|=O\left(h^{4 r}\right) \text { and } \hat{\delta}\left(R(P), R\left(P_{n}^{M}\right)\right)=O\left(h^{3 r}\right),
$$

where $\hat{\delta}$ denotes the gap between the subspaces.

We further consider the case when $\lambda$ is a simple eigenvalue of $T$. Let

$$
T_{n}^{M} \phi_{n}^{M}=\lambda_{n}^{M} \phi_{n}^{M}, \quad\left\|\phi_{n}^{M}\right\|=1,
$$

and

It is shown that

$$
\psi_{n}^{M}=\frac{1}{\lambda_{n}^{M}} T \phi_{n}^{M}
$$

$$
\left\|P \phi_{n}^{M}-\psi_{n}^{M}\right\|=O\left(h^{4 r}\right) .
$$

The above estimates should be compared with the following known estimates for the collocation and the iterated collocation methods (see Chatelin [4):

$$
\begin{aligned}
& \left|\lambda-\hat{\lambda}_{n}^{C}\right|=\left|\lambda-\hat{\lambda}_{n}^{S}\right|=O\left(h^{2 r}\right), \\
& \hat{\delta}\left(R(P), R\left(P_{n}^{C}\right)\right)=O\left(h^{r}\right), \\
& \hat{\delta}\left(R(P), R\left(P_{n}^{S}\right)\right)=O\left(h^{2 r}\right) .
\end{aligned}
$$

Note that the range of $T_{n}^{M}$ is contained in $X_{n} \cup T\left(X_{n}\right)=Y_{n}$, say. Since the dimension of $X_{n}$ is $n r$, the dimension of $Y_{n}$ is $\leq 2 n r$. While considering the eigenvalue problem for $T_{n}^{M}$, it is enough to consider the restriction of $T_{n}^{M}$ to $Y_{n}$. Thus the eigenvalue problem for $T_{n}^{M}$ can be reduced to a matrix eigenvalue problem of size $2 n r$, whereas the eigenvalue problem for $T_{n}^{C}$ or $T_{n}^{S}$ is equivalent to a matrix eigenvalue problem of size $n r$. In the case of an uniform partition of $[0,1]$, the norm of the partition is $h=\frac{1}{n}$. Thus, in the proposed method, an eigenvalue approximation of the order $\left(\frac{1}{n}\right)^{4 r}$ is obtained by solving an eigenvalue problem of size $2 n r$, whereas in the collocation method, in order to achieve the same order of convergence, it is necessary to solve an eigenvalue problem of size $n^{2} r$. The numerical results given in Section 6 confirm this observation.

It is possible to extend our results in various directions. In $[8]$ we have considered the case of the orthogonal projections. We can also use the new operator $T_{n}^{M}$ for approximate solution of an operator equation. As we have introduced a sequence of finite rank operators converging to $T$ in norm, we can define iterative refinement 
schemes for opertor equations as well as eigenvalue problems, multilevel methods, accelerated spectral approximation and extrapolation using this new operator. It is also possible to choose the interpolation points as Lobatto points. These issues will be studied in future papers.

Here is an outline of the paper. In Section 2 we set the notations and prove some preliminary results. In Section 3 we describe our new method in a general setting and obtain some error estimates for eigenelement approximation. In Section 4 we obtain precise orders of convergence for eigenvalue as well as the spectral subspace approximation of an integral operator with a smooth kernel. The projection operator is chosen to be the interpolatory projection at Gauss points. In Section 5, using an iteration technique, we obtain an eigenvector approximation of the order of $4 r$. In Section 6 we illustrate our results by numerical examples.

\section{Preliminaries}

Let $X$ be a complex Banach space and $\mathrm{BL}(X)$ the space of all bounded linear operators on $X$ along with the operator norm. Let $T: X \rightarrow X$ be a compact linear operator and let $\sigma(T)$ and $\rho(T)$ denote the spectrum and the resolvent set of $T$, respectively. Let $\lambda$ be a nonzero eigenvalue of $T$ with algebraic multiplicity $m$. Let $\epsilon$ be such that $0<\epsilon<\operatorname{dist}(\lambda, \sigma(T) \backslash\{\lambda\})$ and $\Gamma$ the positively oriented circle with center $\lambda$ and radius $\epsilon$. Then $\Gamma \subset \rho(T)$ and

$$
\max _{z \in \Gamma}\left\|(T-z I)^{-1}\right\| \leq C_{1} .
$$

Note that throughout this paper $C_{1}, C_{2}, C_{3}$ and $C_{4}$ are constants, and $C$ denotes a generic constant, independent of $n$. Let

$$
P=-\frac{1}{2 \pi i} \int_{\Gamma}(T-z I)^{-1} d z
$$

the spectral projection associated with $T$ and $\lambda$. Then $\operatorname{rank} P=m$.

For nonzero subspaces $Y$ and $Z$ of $X$, let

$$
\delta(Y, Z)=\sup \{\operatorname{dist}(y, Z): y \in Y,\|y\|=1\} .
$$

Then

$$
\hat{\delta}(Y, Z)=\max \{\delta(Y, Z), \delta(Z, Y)\}
$$

is known as the gap between $Y$ and $Z$. For $S \in \mathrm{BL}(X)$, we denote by $R(S)$ the range space. Let $\delta=\min \{|z|: z \in \Gamma\}>0$.

Let $T_{n}$ be a sequence in BL converging to $T$ in collectively compact fashion. We quote the following results from Osborn 9 .

For all large $n, \Gamma \subset \rho\left(T_{n}\right)$ and $\max \left\{\left\|\left(T_{n}-z I\right)^{-1}\right\|: z \in \Gamma\right\} \leq C_{2}$. As the spectral projection

$$
P_{n}=-\frac{1}{2 \pi i} \int_{\Gamma}\left(T_{n}-z I\right)^{-1} d z
$$

is of rank $m$, the spectrum of $T_{n}$ inside $\Gamma$ consists of $m$ eigenvalues $\lambda_{n, 1}, \ldots, \lambda_{n, m}$, counted according to their algebraic multiplicities. Let

$$
\hat{\lambda}_{n}=\frac{\lambda_{n, 1}+\cdots+\lambda_{n, m}}{m}
$$

denote their arithmetic mean. 
Theorem 2.1 (Osborn 9]). For all large n,

$$
\hat{\delta}\left(R(P), R\left(P_{n}\right)\right) \leq C\left\|\left(T-T_{n}\right) T\right\| .
$$

Below we prove a modified version of Theorem 2 of Osborn 9 .

Theorem 2.2. For all large n,

$$
\left|\lambda-\hat{\lambda}_{n}\right| \leq C\left\|T_{n}\left(T-T_{n}\right) T\right\| .
$$

Proof. Let $A_{n}=P_{n \mid R(P)}: R(P) \rightarrow R\left(P_{n}\right)$. The argument given in Theorem 2 of [9] shows that $A_{n}$ is bijective and $\left\|A_{n}^{-1}\right\| \leq 2$ for all large $n$. Define $\hat{T}=T_{\mid R(P)}$ and $\hat{T}_{n}=A_{n}^{-1} T_{n} A_{n}$. Then

$$
\begin{aligned}
\left|\lambda-\hat{\lambda}_{n}\right| & =\frac{1}{m}\left|\operatorname{trace}\left(\hat{T}-\hat{T}_{n}\right)\right| \leq\left\|\hat{T}-\hat{T}_{n}\right\| \\
& =\sup _{n}\left\{\left\|A_{n}^{-1} P_{n}\left(T-T_{n}\right) x\right\|: x \in R(P),\|x\|=1\right\} \\
& \leq 2\left\|P_{n}\left(T-T_{n}\right) P\right\| .
\end{aligned}
$$

Using the integral representations of $P$ and $P_{n}$, it can be seen that

$$
\left\|P_{n}\left(T-T_{n}\right) P\right\| \leq\left(\frac{\epsilon}{\delta}\right)^{2} C_{1} C_{2}\left\|T_{n}\left(T-T_{n}\right) T\right\|,
$$

which proves the result.

\section{A NEW PROJECTION METHOD}

Let $\pi_{n}: X \rightarrow X$ be a sequence of bounded projections such that for each $x \in X$, $\pi_{n} x \rightarrow x$ as $n \rightarrow \infty$ and $X_{n}=R\left(\pi_{n}\right)$ is finite dimensional.

We propose to approximate

$$
T \phi=\lambda \phi
$$

by

$$
\left(\pi_{n} T \pi_{n}+\pi_{n} T\left(I-\pi_{n}\right)+\left(I-\pi_{n}\right) T \pi_{n}\right) \phi_{n}^{M}=\lambda_{n}^{M} \phi_{n}^{M} ;
$$

that is,

$$
T_{n}^{M} \phi_{n}^{M}=\lambda_{n}^{M} \phi_{n}^{M} .
$$

Since $T_{n}^{M}$ converges to $T$ in the norm, for all large $n, T_{n}^{M}$ has $m$ eigenvalues $\lambda_{n, 1}^{M}, \ldots, \lambda_{n, m}^{M}$ inside $\Gamma$. Let $\hat{\lambda}_{n}^{M}$ denote the arithmetic mean of these $m$ eigenvalues and let $P_{n}^{M}$ denote the associated spectral projection. Then the following result follows from Theorems 2.1 and 2.2 .

Theorem 3.1. For all large $n$

$$
\begin{aligned}
& \hat{\delta}\left(R(P), R\left(P_{n}^{M}\right)\right) \leq C\left\|\left(I-\pi_{n}\right) T\left(I-\pi_{n}\right) T\right\|, \\
& \left|\lambda-\hat{\lambda}_{n}^{M}\right| \leq C\left\|T\left(I-\pi_{n}\right) T\left(I-\pi_{n}\right) T\right\| .
\end{aligned}
$$

Let $\hat{\lambda}_{n}^{C}=\hat{\lambda}_{n}^{S}$ denote the arithmetic mean of $m$ eigenvalues of $T_{n}^{C}=\pi_{n} T \pi_{n}$ in the Galerkin method or $T_{n}^{S}=T \pi_{n}$ in the Sloan method, approximating eigenvalue $\lambda$ of $T$. Let $P_{n}^{C}$ and $P_{n}^{S}$ denote the spectral projections associated with $T_{n}^{C}$ and $T_{n}^{S}$, respectively. Then the following result follows from Theorems 2.1 and 2.2 . 
Theorem 3.2. For all large $n$

$$
\begin{aligned}
& \hat{\delta}\left(R(P), R\left(P_{n}^{C}\right)\right) \leq C\left\|\left(T-\pi_{n} T \pi_{n}\right) T\right\|, \\
& \hat{\delta}\left(R(P), R\left(P_{n}^{S}\right)\right) \leq C\left\|T\left(I-\pi_{n}\right) T\right\|, \\
& \left|\lambda-\hat{\lambda}_{n}^{C}\right|=\left|\lambda-\hat{\lambda}_{n}^{S}\right| \leq C\left\|T\left(I-\pi_{n}\right) T\right\| .
\end{aligned}
$$

A comparison of bounds (3.2)-(3.3) and (3.4) $-(3.6)$ suggests that the eigenelement approximation using $T_{n}^{M}$ may be better than Galerkin or Sloan approximation. In the next section it is shown that, in fact, the order of convergence is doubled by using the new operator.

\section{Orders of CONVERGENCE}

Let $X=C[0,1]$ with the supremum norm. Choose $r \geq 1$ and assume that $k(.,.) \in C^{2 r}([0,1] \times[0,1])$.

Consider the integral operator

$$
(T u)(s)=\int_{0}^{1} k(s, t) u(t) d t, \quad s \in[0,1] .
$$

Then $T: C[0,1] \rightarrow C[0,1]$ is a compact linear operator. In fact, $R(T) \subset C^{2 r}[0,1]$. For $u \in C^{2 r}[0,1], u^{(2 r)}$ denotes the $2 r$-th derivative of $u$. We set

$$
\begin{aligned}
D^{i, j} k(s, t) & =\frac{\partial^{i+j}}{\partial s^{i} \partial t^{j}} k(s, t), \quad s, t \in[0,1] \\
\|k\|_{2 r, \infty} & =\sum_{i=0}^{2 r} \sum_{j=0}^{2 r}\left\|D^{i, j} k\right\|_{\infty}
\end{aligned}
$$

and

$$
\|u\|_{2 r, \infty}=\sum_{i=0}^{2 r}\left\|u^{(i)}\right\|_{\infty}
$$

Consider a partition

$$
0=t_{0}<t_{1}<\cdots<t_{n}=1
$$

of $[0,1]$ and for $j=1, \ldots, n$, set $h_{j}=t_{j}-t_{j-1}, \quad h=\max \left\{h_{j}: j=1, \ldots, n\right\}$. We assume that $h \rightarrow 0$ as $n \rightarrow \infty$. Let $X_{n}$ be the space of all piecewise polynomials of order $r$ (i.e., of degree $\leq r-1$ ) with breakpoints at $t_{1}, \ldots, t_{n-1}$. We impose no continuity conditions at the breakpoints.

Let $B_{r}=\left\{\tau_{1}, \ldots, \tau_{r}\right\}$ denote the set of $r$ Gauss points, i.e., the zeros of the (Legendre) polynomial $\frac{d^{r}}{d s^{r}}\left(s^{2}-1\right)^{r}$ in the interval $[-1,1]$.

Define $f_{j}:[-1,1] \rightarrow\left[t_{j-1}, t_{j}\right]$ as

$$
f_{j}(t)=\frac{1-t}{2} t_{j-1}+\frac{1+t}{2} t_{j}, \quad t \in[-1,1] .
$$

Let $A=\bigcup_{j=1}^{n} f_{j}\left(B_{r}\right)$, the set of $n r$ Gauss points.

The map $\pi_{n}: C[0,1] \rightarrow X_{n}$ is defined by

$$
\pi_{n} u \in X_{n},\left(\pi_{n} u\right)(t)=u(t), t \in A .
$$

Then $\pi_{n} u \rightarrow u$ as $n \rightarrow \infty$ for each $u \in C[0,1]$ and the results of Section 3 are applicable. Note that $\pi_{n} u$ is, in general, discontinuous at the breakpoints.

In what follows we use crucially the following two estimates. 
For $u \in C^{r}[0,1]$ (see Chatelin [4]),

$$
\left\|\left(I-\pi_{n}\right) u\right\|_{\infty} \leq C_{3}\left\|u^{(r)}\right\|_{\infty} h^{r} .
$$

Let $f \in C^{r}[0,1]$ and $g \in C^{2 r}[0,1]$. Then (see de-Boor-Swartz [7])

$$
\left|\int_{0}^{1} f(t)\left(I-\pi_{n}\right) g(t) d t\right| \leq C_{4}\|f\|_{r, \infty}\|g\|_{2 r, \infty} h^{2 r} .
$$

Theorem 4.1. If $\pi_{n}: C[0,1] \rightarrow X_{n}$ is the interpolatory projection defined above and $T$ is an integral operator with kernel $\left.k(\cdot, \cdot) \in C^{2 r}([0,1]) \times[0,1]\right)$, then

$$
\begin{gathered}
\left\|\left(I-\pi_{n}\right) T\right\|=O\left(h^{r}\right), \\
\left\|T\left(I-\pi_{n}\right) T\right\|=O\left(h^{2 r}\right), \\
\left\|\left(I-\pi_{n}\right) T\left(I-\pi_{n}\right) T\right\|=O\left(h^{3 r}\right), \\
\left\|T\left(I-\pi_{n}\right) T\left(I-\pi_{n}\right) T\right\|=O\left(h^{4 r}\right) .
\end{gathered}
$$

Proof. Let $u \in C[0,1]$. Since for $s \in[0,1]$ and $i=0,1 \ldots, 2 r$,

$$
(T u)^{(i)}(s)=\int_{0}^{1} \frac{\partial^{i}}{\partial s^{i}} k(s, t) u(t) d t
$$

it follows that

$$
\left\|(T u)^{(i)}\right\|_{\infty} \leq\left\|D^{i, 0} k\right\|_{\infty}\|u\|_{\infty}
$$

and

$$
\|T u\|_{2 r, \infty} \leq\|k\|_{2 r, \infty}\|u\|_{\infty}
$$

Then by (4.1)

$$
\left\|\left(I-\pi_{n}\right) T u\right\|_{\infty} \leq C_{3}\left\|(T u)^{(r)}\right\|_{\infty} h^{r} \leq C_{3}\left\|D^{r, 0} k\right\|_{\infty}\|u\|_{\infty} h^{r},
$$

which proves (4.3).

Since

$$
\left(T\left(I-\pi_{n}\right) T u\right)(s)=\int_{0}^{1} k(s, t)\left(I-\pi_{n}\right)(T u)(t) d t,
$$

by (4.2) and (4.7),

$$
\begin{aligned}
\left\|T\left(I-\pi_{n}\right) T u\right\|_{\infty} & \leq C_{4}\|k\|_{r, \infty}\|T u\|_{2 r, \infty} h^{2 r} \\
& \leq C_{4}\|k\|_{r, \infty}\|k\|_{2 r, \infty}\|u\|_{\infty} h^{2 r},
\end{aligned}
$$

which proves (4.4).

For $i=0,1 \ldots, 2 r$,

$$
\left(T\left(I-\pi_{n}\right) T u\right)^{(i)}(s)=\int_{0}^{1} \frac{\partial^{i}}{\partial s^{i}} k(s, t)\left(I-\pi_{n}\right)(T u)(t) d t .
$$

Hence by (4.2),

$$
\left|\left(T\left(I-\pi_{n}\right) T u\right)^{(i)}(s)\right| \leq C_{4}\left(\sum_{j=0}^{r}\left\|D^{i, j} k\right\|_{\infty}\right)\|T u\|_{2 r, \infty} h^{2 r} .
$$


As a consequence, using (4.7), we get

$$
\left\|\left(T\left(I-\pi_{n}\right) T u\right)^{(r)}\right\|_{\infty} \leq C_{4}\|k\|_{r, \infty}\|k\|_{2 r, \infty}\|u\|_{\infty} h^{2 r}
$$

and

$$
\left\|T\left(I-\pi_{n}\right) T u\right\|_{2 r, \infty} \leq C_{4}\left(\|k\|_{2 r, \infty}\right)^{2}\|u\|_{\infty} h^{2 r} .
$$

Next, by (4.1) and (4.9),

$$
\begin{aligned}
\left\|\left(I-\pi_{n}\right) T\left(I-\pi_{n}\right) T u\right\|_{\infty} & \leq C_{3}\left\|\left(T\left(I-\pi_{n}\right) T u\right)^{(r)}\right\|_{\infty} h^{r} \\
& \leq C_{3} C_{4}\|k\|_{r, \infty}\|k\|_{2 r, \infty}\|u\|_{\infty} h^{3 r}
\end{aligned}
$$

which proves (4.5).

Lastly, by (4.8) and (4.10),

$$
\begin{aligned}
\left\|T\left(I-\pi_{n}\right) T\left(I-\pi_{n}\right) T u\right\|_{\infty} & \leq C_{4}\|k\|_{r, \infty}\left\|T\left(I-\pi_{n}\right) T u\right\|_{2 r, \infty} h^{2 r} \\
& \leq\left(C_{4}\right)^{2}\|k\|_{r, \infty}\left(\|k\|_{2 r, \infty}\right)^{2}\|u\|_{\infty} h^{4 r},
\end{aligned}
$$

which completes the proof.

Combining the results of Theorems 3.1 and 4.1 we obtain the following orders of convergence for eigenvalue and spectral subspace approximation using the new method.

Theorem 4.2. For all large $n$,

$$
\begin{aligned}
\hat{\delta}\left(R(P), R\left(P_{n}^{M}\right)\right) & =O\left(h^{3 r}\right), \\
\left|\lambda-\hat{\lambda}_{n}^{M}\right| & =O\left(h^{4 r}\right) .
\end{aligned}
$$

Also, from Theorems 3.2 and 4.1 we obtain the following orders of convergence for the collocation and the iterated collocation methods. These orders of convergence are well known (see, for example, Chatelin [4]):

$$
\begin{aligned}
\hat{\delta}\left(R(P), R\left(P_{n}^{C}\right)\right) & =O\left(h^{r}\right), \\
\hat{\delta}\left(R(P), R\left(P_{n}^{S}\right)\right) & =O\left(h^{2 r}\right), \\
\left|\lambda-\hat{\lambda}_{n}^{C}\right| & =\left|\lambda-\hat{\lambda}_{n}^{S}\right|=O\left(h^{2 r}\right) .
\end{aligned}
$$

A comparison of (4.11)-(4.12) and (4.13)-(4.15) show that the order of convergence $h^{2 r}$ for eigenvalue approximation in the collocation/iterated collocation method is improved to $h^{4 r}$ in the new method. For spectral subspaces the improvement is from $h^{r}$ in the collocation method and from $h^{2 r}$ in the iterated collocation method to $h^{3 r}$ in the new method. In Section 6 we illustrate the above results by numerical examples. In the next section we show that in the case of a simple eigenvalue, the order of convergence for eigenvector approximation can be further improved to $h^{4 r}$ by using an iteration technique.

\section{IMPROVEMENT BY ITERATION}

In this section we restrict ourselves to the case when $\lambda$ is a simple eigenvalue. Let

We define

$$
T_{n}^{M} \phi_{n}^{M}=\lambda_{n}^{M} \phi_{n}^{M}, \quad\left\|\phi_{n}^{M}\right\|=1 .
$$

$$
\psi_{n}^{M}=\frac{1}{\lambda_{n}^{M}} T \phi_{n}^{M} .
$$


Let

$$
\phi=P \phi_{n}^{M} .
$$

Theorem 5.1. For all large $n$

$$
\left\|\phi-\psi_{n}^{M}\right\|=O\left(h^{4 r}\right) .
$$

Proof. Recall that $\Gamma$ is a circle with center $\lambda$ and radius $\epsilon$. Choose $n$ big enough so that $\left|\lambda-\lambda_{n}^{M}\right| \leq \frac{\epsilon}{2}$. Then since $|\lambda|>\epsilon$, we have $\left|\lambda_{n}^{M}\right|>\frac{\epsilon}{2}$ and for $z \in \Gamma$, $\left|z-\lambda_{n}^{M}\right|>\frac{\epsilon}{2}$. Consider

$$
\begin{aligned}
\phi-\psi_{n}^{M} & =\frac{1}{\lambda} T \phi-\frac{1}{\lambda_{n}^{M}} T \phi_{n}^{M} \\
& =\left(\frac{1}{\lambda}-\frac{1}{\lambda_{n}^{M}}\right) T \phi+\frac{1}{\lambda_{n}^{M}} T\left(\phi-\phi_{n}^{M}\right) .
\end{aligned}
$$

Now

$$
\left\|\left(\frac{1}{\lambda}-\frac{1}{\lambda_{n}^{M}}\right) T \phi\right\| \leq \frac{\left|\lambda-\lambda_{n}^{M}\right|\|P\|}{\left|\lambda_{n}^{M}\right|} \leq \frac{2}{\epsilon}\|P\|\left|\lambda-\lambda_{n}^{M}\right| \leq C h^{4 r}
$$

by (4.12).

Next

$$
\begin{aligned}
T\left(\phi-\phi_{n}^{M}\right) & =T\left(P-P_{n}^{M}\right) \phi_{n}^{M} \\
& =-\frac{1}{2 \pi i} \int_{\Gamma} T\left((T-z I)^{-1}-\left(T_{n}^{M}-z I\right)^{-1}\right) \phi_{n}^{M} d z \\
& =\frac{1}{2 \pi i} \int_{\Gamma} T(T-z I)^{-1}\left(T-T_{n}^{M}\right)\left(T_{n}^{M}-z I\right)^{-1} \phi_{n}^{M} d z \\
& =\frac{1}{2 \pi i} \int_{\Gamma} \frac{(T-z I)^{-1} T\left(I-\pi_{n}\right) T\left(I-\pi_{n}\right) \phi_{n}^{M}}{\lambda_{n}^{M}-z} d z \\
& =\frac{1}{2 \pi i} \int_{\Gamma} \frac{(T-z I)^{-1} T\left(I-\pi_{n}\right) T\left(I-\pi_{n}\right) T \pi_{n} \phi_{n}^{M}}{\lambda_{n}^{M}\left(\lambda_{n}^{M}-z\right)} d z .
\end{aligned}
$$

Hence

and by $(4.6)$

$$
\left\|T\left(\phi-\phi_{n}^{M}\right)\right\| \leq \frac{4}{\epsilon} C_{1}\left\|\pi_{n}\right\|\left\|T\left(I-\pi_{n}\right) T\left(I-\pi_{n}\right) T\right\|
$$

$$
\left\|T\left(\phi-\phi_{n}^{M}\right)\right\|=O\left(h^{4 r}\right) .
$$

The result now follows by combining (5.2) and the above estimate.

\section{NUMERICAL EXAMPLES}

We consider the integral operator $T$ given by

$$
(T u)(s)=\int_{0}^{1} \exp (s t) u(t) d t, \quad s \in[0,1]
$$

In actual computation $T$ is replaced by its approximation $\tilde{T}$ given by

$$
\tilde{T} u(s)=\sum_{j=1}^{m} w_{j}^{(m)} \exp \left(s t_{j}^{(m)}\right) u\left(t_{j}^{(m)}\right), \quad u \in C[0,1], \quad s \in[0,1],
$$

where $m$ is very large. 
Here the nodes $t_{1}^{(m)}, \ldots, t_{m}^{(m)}$ in $[0,1]$ and the weights $w_{1}^{(m)}, \ldots, w_{m}^{(m)}$ in $\mathbb{C}$ give a convergent quadrature formula

$$
Q u=\sum_{j=1}^{m} w_{j}^{(m)} u\left(t_{j}^{(m)}\right), \quad x \in C[0,1] .
$$

Collocation at Gauss Points. We choose $X_{n}$ to be the space of piecewise constant functions $(r=1)$ or the space of piecewise linear functions $(r=2)$ with respect to the equidistant partition

$$
0<\frac{1}{n}<\frac{2}{n}<\cdots<\frac{n}{n}=1 .
$$

The collocation points are either midpoints

$$
t_{j}^{(n)}=\frac{2 j-1}{n}, j=1, \ldots, n
$$

or Gauss 2 points

$$
t_{j}^{(n)}= \begin{cases}\frac{j-\frac{1}{\sqrt{3}}}{n}, & \text { if } j \text { is odd, } \\ \frac{j-1+\frac{1}{\sqrt{3}}}{n}, & \text { if } j \text { is even, }\end{cases}
$$

$j=1, \ldots, n$.

The projection $\pi_{n}: C[0,1] \rightarrow X_{n}$ is the interpolatory projection.

We choose

$$
w_{j}^{(m)}=\frac{1}{m}, \quad t_{j}^{(m)}=\frac{2 j-1}{m}, \quad j=1, \ldots, m,
$$

when $r=1$ and

$$
w_{j}^{(m)}=\frac{1}{m}, t_{j}^{(m)}= \begin{cases}\frac{j-\frac{1}{\sqrt{3}}}{m}, & \text { if } j \text { is odd } \\ \frac{j-1+\frac{1}{\sqrt{3}}}{m}, & \text { if } j \text { is even }\end{cases}
$$

when $r=2$.

We fix $m=512$. For $r=1$, we choose $n=4,8,16,32,64$ and for $r=2$, we choose $n=2,4,8,16$.

Let $\tilde{\lambda}$ be the largest eigenvalue of $\tilde{T}$, in modulus, and let $\lambda_{n}^{M}, \lambda_{n}^{C}$ be the eigenvalues obtained by using the new method and the collocation method, respectively. Let $\phi_{n}^{M}$ and $\phi_{n}^{C}$ be the associated eigenvectors.

We write

$$
\begin{aligned}
& \left|\tilde{\lambda}-\lambda_{n}^{C}\right| \simeq K_{1} h^{\alpha}, \quad\left|\tilde{\lambda}-\lambda_{n}^{M}\right| \simeq K_{2} h^{\beta}, \\
& \left\|\tilde{P} \phi_{n}^{C}-\phi_{n}^{C}\right\| \leq K_{3} h^{\gamma}, \quad\left\|\tilde{P} \phi_{n}^{M}-\phi_{n}^{M}\right\| \simeq K_{4} h^{\eta} .
\end{aligned}
$$

Since $h=\frac{1}{n}$, we use two successive values of $n$ to determine $\alpha, \beta, \gamma$ and $\eta$.

In Table 6.1 we give the error in the eigenvalue approximation and the computed values of $\alpha$ and $\beta$ in the collocation/iterated collocation method at midpoints and the new method. Note that the theoretically predicted values are $\alpha=2, \beta=4$.

In Table 6.2 the errors in the eigenvector approximation and the computed orders of convergence $\gamma$ and $\eta$ in the collocation at midpoints and the new method are given. Note that the theoretically predicted values are $\gamma=1, \eta=3$. 
TABLE 6.1. Collocation at midpoint $(r=1)$. Theoretically predicted values: $\alpha=2, \beta=4$

\begin{tabular}{|l|c|c|c|c|}
\hline$n$ & $\left|\lambda-\lambda_{n}^{C}\right|$ & $\left|\lambda-\lambda_{n}^{M}\right|$ & $\alpha$ & $\beta$ \\
\hline 4 & $4.00 \times 10^{-3}$ & $3.81 \times 10^{-5}$ & & \\
8 & $1.01 \times 10^{-3}$ & $2.42 \times 10^{-6}$ & 1.99 & 3.98 \\
16 & $2.52 \times 10^{-4}$ & $1.52 \times 10^{-7}$ & 2.00 & 4.00 \\
32 & $6.28 \times 10^{-5}$ & $9.42 \times 10^{-9}$ & 2.01 & 4.01 \\
64 & $1.54 \times 10^{-5}$ & $5.75 \times 10^{-10}$ & 2.02 & 4.03 \\
\hline
\end{tabular}

TABLE 6.2. Collocation at midpoint $(r=1)$. Theoretically predicted values: $\gamma=1, \eta=3$

\begin{tabular}{|l|c|c|c|c|}
\hline$n$ & $\left\|P \phi_{n}^{C}-\phi_{n}^{C}\right\|$ & $\left\|P \phi_{n}^{M}-\phi_{n}^{M}\right\|$ & $\gamma$ & $\eta$ \\
\hline 4 & $4.75 \times 10^{-2}$ & $3.44 \times 10^{-4}$ & & \\
8 & $1.73 \times 10^{-2}$ & $3.19 \times 10^{-5}$ & 1.46 & 3.43 \\
16 & $6.13 \times 10^{-3}$ & $2.84 \times 10^{-6}$ & 1.56 & 3.49 \\
32 & $2.11 \times 10^{-3}$ & $2.45 \times 10^{-7}$ & 1.53 & 3.53 \\
64 & $7.01 \times 10^{-4}$ & $2.01 \times 10^{-8}$ & 1.59 & 3.61 \\
\hline
\end{tabular}

TABle 6.3. Collocation at Gauss 2 points $(r=2)$. Theoretically predicted values: $\alpha=4, \beta=8$

\begin{tabular}{|l|c|c|c|c|}
\hline$n$ & $\left|\lambda-\lambda_{n}^{C}\right|$ & $\left|\lambda-\lambda_{n}^{M}\right|$ & $\alpha$ & $\beta$ \\
\hline 2 & $4.30 \times 10^{-5}$ & $2.35 \times 10^{-8}$ & & \\
4 & $2.73 \times 10^{-6}$ & $9.93 \times 10^{-11}$ & 3.98 & 7.88 \\
8 & $1.71 \times 10^{-7}$ & $3.98 \times 10^{-13}$ & 3.99 & 7.96 \\
16 & $1.07 \times 10^{-8}$ & $4.44 \times 10^{-15}$ & 4.00 & 6.49 \\
\hline
\end{tabular}

TABLE 6.4. Collocation at Gauss 2 points $(r=2)$. Theoretically predicted values: $\gamma=2, \eta=6$

\begin{tabular}{|l|c|c|c|c|}
\hline$n$ & $\left\|P \phi_{n}^{C}-\phi_{n}^{C}\right\|$ & $\left\|P \phi_{n}^{M}-\phi_{n}^{M}\right\|$ & $\gamma$ & $\eta$ \\
\hline 2 & $5.56 \times 10^{-3}$ & $1.90 \times 10^{-6}$ & & \\
4 & $1.04 \times 10^{-3}$ & $2.40 \times 10^{-8}$ & 2.42 & 6.30 \\
8 & $1.86 \times 10^{-4}$ & $2.78 \times 10^{-10}$ & 2.48 & 6.43 \\
16 & $3.20 \times 10^{-5}$ & $3.04 \times 10^{-12}$ & 2.54 & 6.52 \\
\hline
\end{tabular}

The corresponding results for collocation at Gauss 2 points are listed in Tables 6.3 and 6.4. In this case the theoretically predicted values are $\alpha=4, \beta=8, \gamma=$ $2, \eta=6$. 
Note from Table 6.1 that both the errors $\left|\lambda-\lambda_{4}^{M}\right|$ and $\left|\lambda-\lambda_{64}^{C}\right|$ are of the order of $10^{-5}$. The computation of $\lambda_{4}^{M}$ needs the solution of a matrix eigenvalue problem of size 8 whereas $\lambda_{64}^{C}$ is obtained by solving a matrix eigenvalue problem of size 64 . Also $\lambda_{32}^{M}$, which is obtained by solving a matrix eigenvalue problem of size 64 , has error of the order of $10^{-8}$. Similar observations can be made from Tables 6.2 6.4.

In Tables 6.1 and 6.3 the observed values of $\alpha$ and $\beta$ match well with the theoretically predicted values. In the case of the collocation at midpoints, the expected values of $\gamma$ and $\eta$ are 1 and 3, respectively, whereas their observed values are about 1.5 and 3.5. Similarly, in the case of the collocation at Gauss 2 points, the expected values of $\gamma$ and $\eta$ are 2 and 6 , respectively, whereas their observed values are about 2.5 and 6.5. The theoretically predicted values for $\gamma$ and $\eta$ are obtained by using an upper bound for the error in the eigenvector approximation, and the error seems to converge faster than the upper bound.

\section{REFERENCES}

1. K. Atkinson, The Numerical Solution of Integral Equations of the Second Kind, Cambridge University Press, 1997. MR1464941 (99d:65364)

2. K. Atkinson, I. Graham and I. Sloan, Piecewise continuous collocation for integral equations, SIAM J. of Numerical Analysis, 20 (1983), pp. 172-186. MR0687375 (85a:65175)

3. C.T.H. Baker, The Numerical Treatment of Integral Equations, Oxford University Press, Oxford, 1977. MR 0467215 (57:7079)

4. F. Chatelin, Spectral Approximation of Linear Operators, Academic Press, New York, 1983. MR0716134 (86d:65071)

5. F. Chatelin and R. Lebbar, Superconvergence results for the iterated projection method applied to a Fredholm integral equation of the second kind and the corresponding eigenvalue problem, J. Integral Equations, 6 (1984), pp. 71-91. MR0727937 (85i:65167)

6. C. de Boor and B. Swartz, Collocation at Gaussin points, SIAM J. Numer. Anal., 10 (1973), pp. 582-606. MR0373328 (51:9528)

7. C. de Boor Aand B. Swartz, Collocation approximation to eigenvalues of an ordinary differential equation: The principle of the thing, Math. Comp., 35 (1980), 679-694. MR0572849 (81k:65097)

8. R. P. Kulkarni, A New Superconvergent Projection Method for Approximate Solutions of Eigenvalue Problems, Numerical Functional Analysis and Optimization, 24 (2003), 75-84. MR.1978953 (2004b:45003)

9. J. E. Osborn, Spectral Approximation for Compact operators, Math. Comp., 29 (1975), 712725. MR0383117(52:3998)

10. I. H. Sloan, Superconvergence, Numerical Solution of Integral Equations (M. Golberg, ed.), Plenum Press (1990), pp. 35-70. MR1067150 (91g:45011)

Department of Mathematics, Indian Institute of Technology, Powai, Mumbai 400076 , INDIA

E-mail address: rpk@math.iitb.ac.in 